\title{
Embedding and Extracting the Watermark in Video Files using Intelligent Techniques
}

\author{
Shahba I. Khaleel \\ College of Computer Science and Mathematics \\ University of Mosul, Mosul, Iraq
}

Received on: 08/08/2010

ABSTRACT

Accepted on: 21/12/2010

This research, design a watermarking system for embedding and extracting the watermark in video files. This research presented four-efficient and powerful ways to embed and extract the watermark, the extract which in turn characterized as blind since it does not need the original cover in the process of extracting the watermark. The first method established the mean and SVD, which relied on the conversion of Singular values decomposition SVD and calculate the average of the unique values which are resulting from the application of the SVD on each data frame of the video file that required for embedding by, and then this method has been improved by hybrid intelligence by using a genetic algorithm for embedding the watermark, which is a second method. And other two methods has been implemented in this research. The third and fourth methods use artificial neural networks to embed the watermark. Depending on the characteristics of the data frame we use elman and Jordan neural networks and we use genetic algorithm to generate the secret key. The four methods of watermark were efficient and robust against various attacks which was found by measuring the efficiency of the methods by calculating the values of the Peak Signal-to-Noise Ratio PSNR and the correlation coefficient Normalized Correlation Coefficient NCC.

Keywords: watermarking, genetic algorithm, artificial neural networks.

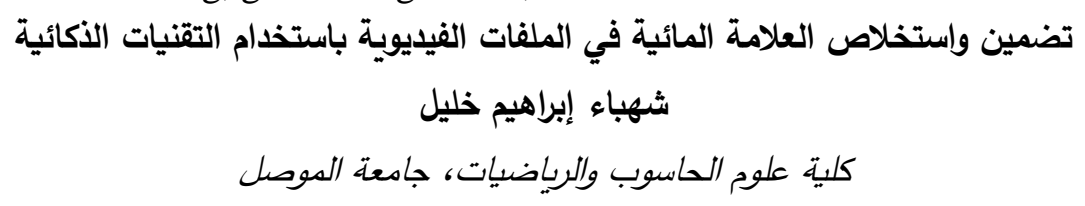

تاريخ قبول البحث: 21 | 12 | 2010

تاريخ استلام البحث: 8 / 8 / 8010

\footnotetext{
الملخص

تم في هذا البحث تصميم نظام للعلامة المائية لتضمين واستخلاص العلامة المائية في الملفات الفيديوية حيث

قدم هذا البحث أربع طرائق كفوهة وقوية لتضمين العلامة المائية واستخلاصها والذي بدوره اتصف بالأعمى لعدم الحاجة

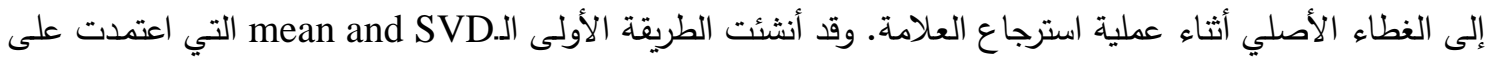

تحويل Singular values decomposition SVD وحساب المعدل للقيم الفريدة (للاستفادة منه بالتضمين) الناتجة

من تطبيق الـSVD على بيانات كل إطار من إطارات الملف الفيديوي المطلوب التضمين فيه. ومن ثم تم تحسين هذه

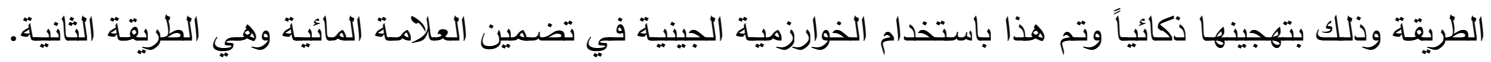

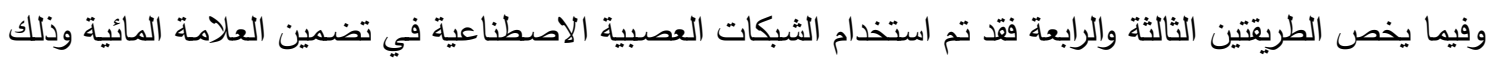

بالاعتماد على خصائص بيانات الإطار باستخدام شبكتي (Jordan و (Jonan) العصبية واستخدام الخوارزمية الجينية

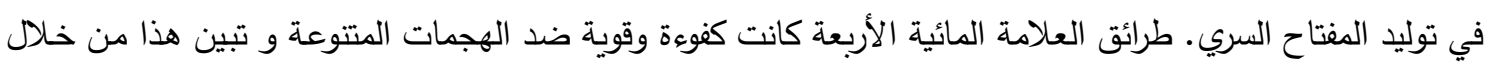

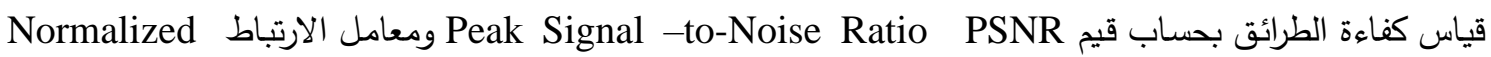

.Correlation Coefficient NCC

الكلمات المفتاحية: العلامة المائية، الخوارزمية الجينية، الشبكة العصبية الاصطناعية. 
ظهرت العلامات المائية الورقية في فن الصناعة اليدويـة قبل 700 سنة تقريبا، حيث وجدت أقدم ورقة

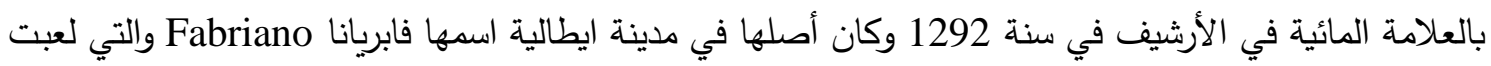
دوراً رئيسياً في تطور الصناعة الورقية، حيث كانت العلامة المائية هي الطريقة المثالية للقضاء على الئي التئية احتمالية

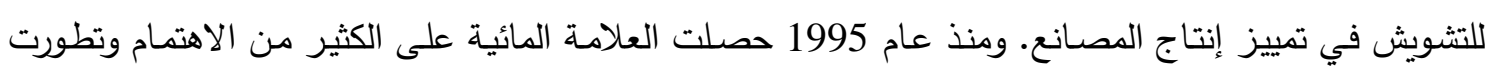
بسرعة كبيرة، حيث توجد العديد من المواضيع المفتوحة لبحوث أكثر تطوراً.

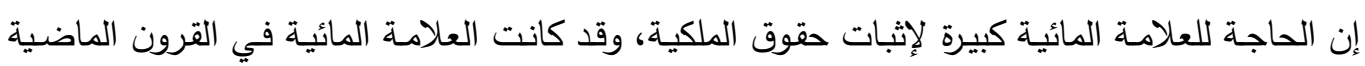
محسوسة على شكل طوابع، تواقيع ونحو ذلك. ومع التطور الذي حصل في جانب الحاسوب ومعالجة الصور فان

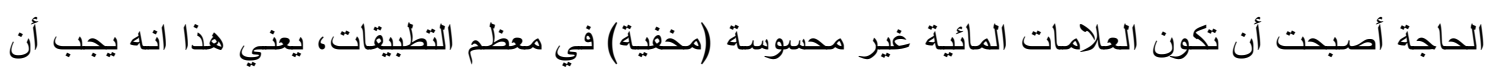
يكون من الصعب استخلاص أو إزالة العلامة المائية من المادة المؤشرة مائيا.

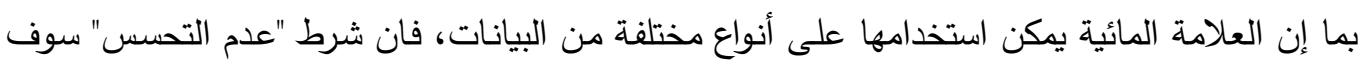

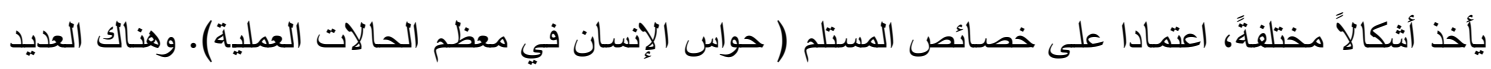
من المصطلحات المستخدمة في العلامات المائية الرقمية والتي لا بد من إيضاحس الإنسان ففهومها: - فالعلامة المائية هي شيء غير منظور يضاف إلى إثارة الغطاء من اجل إخفاء البيانات المخبأة وتكون العلامـة

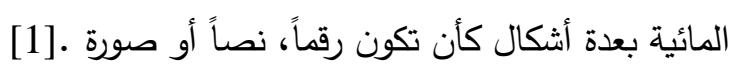
- التأشير المـائي هي عملية تضمين معلومات في مادة إثـارة أخرى. ويكون إدخال هذا النظام (نظام التأشير

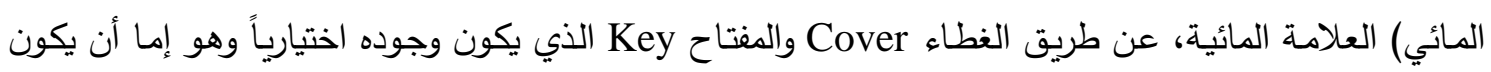

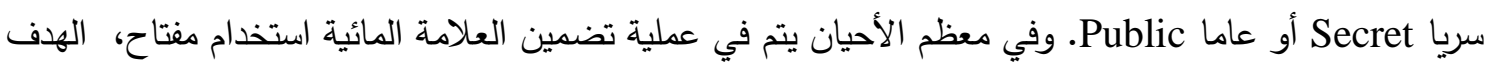

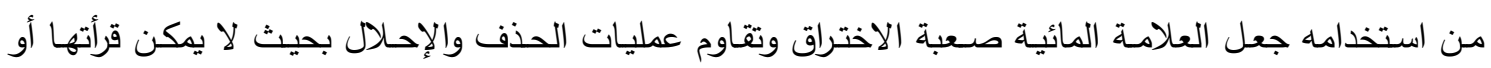
تغييرها بدون استخدام المفتاح نفسه. أما الإخراج فيكون بيانات الغطاء بعد تضمين العلامة المائية فيه [2] . يهدف هذا البحث إلى تصميم نظام لتضمين واستخلاص العلامـة المائية بالملفات الفيديويـة باستخدام

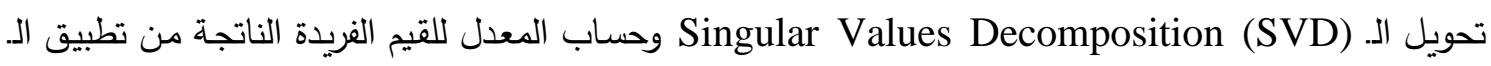
على بيانات كل إطار من إطارات الملف الفيديوي المطلوب التضمين فيه ومن ثم تحسين هذه الطريقة وذلك

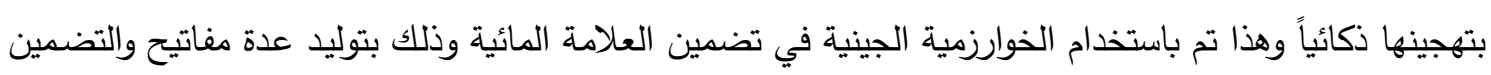
بها ومن ثم تعريض الغطاء المتضمن للعلامة للعديد من الهجمات ومن ثم حساب دالة اللياقة المعتمدة على معامل الارتباط ومقياس جودة الغطاء Peak Signal -to-Noise Ratio PSNR وبالاعتماد على قيمة دالة اللياقة يتم تحديد المواقع المثلى التي يجب التضمين بها وأيضاً تم في هذا البحث استخدام الثبكات العصبية الاصطناعية في

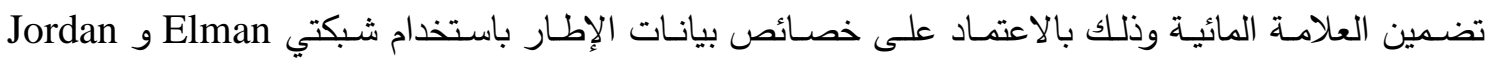
واستخدام الخوارزمية الجينية في توليد المفتاح السري.

2. البحوث السابقة

قدم الباحثون R. A. Ghazy و R. A. El-fishawy وآخرون طريقة كفوءة لتضمين العلامة المائية بالصور الرقمية باستخدام التقنية الرياضية SVD وذلك باعتماد تقطيع الصورة إلى مقاطع غير متداخلة والتضمين

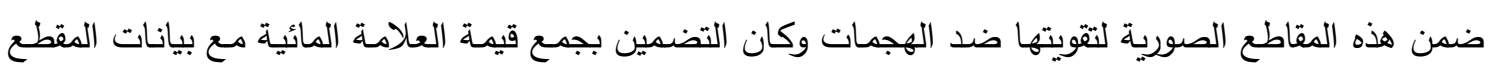


المعين [7]. قدم كل من Maher EL'ARBI ، CHOKRI BEN AMAR و Menri NICOLAS طريقة التضمين العلامـة المائية في الفيديو باستخدام تحويل المويجة المتقطع وتخمين الحركة متعدد الأبعاد

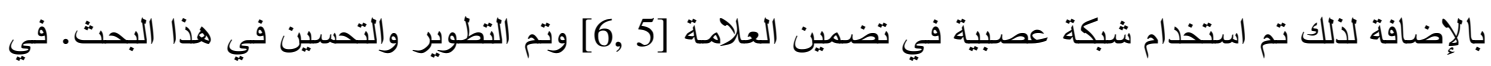
البحث الموسوم "Image Watermarking Based on Genetic Algorithm " تم اقتراح طريقة جديدة لتحسين عمل تضمين العلامة المائية وذلك باعتماد الخوارزمية الجينية وتحويل الجيب تمام المتقطع Discrete Cosine Transform DCT المتقطع لتضمين العلامـة المائية بطريقة الطيف المبعثر فيها وطبق ذلك على الصور وتم الحصول على نتائج

جيدة [16]. اقترح الباحثون Yoo C. Jang D. و Lee S. بحثهم الموسوم" An Svd Based Watermarking Method for Image Content Authentication with Improved Security

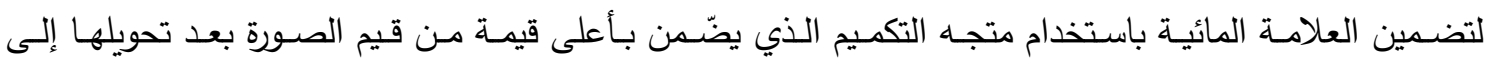
Singular Values Decomposition (SVD) [11]. اقترح الباحثون Shieh C., Huang H., Wang F. and Pan J طريقة جديدة في تضمين العلامة المائية باستخدام تحويل الجيب تمام المتقطع DCT مع الخوارزمية الجينية وذلك بتقسيم الصورة إلى مقاطع حجمها 8*8 وعمل DCT عليها وتكون هذه المقاطع غير متداخلة وتضمين العلامة المائية في هذه المقاطع وتعريضها للهجمات ومن ثم حساب قيمة معامل الارتباط ونسبة تشوه الإثارة واتخاذها كدالة لياقة حيث أن معامل ارتباط كل قيمة منه تعامل مع معامل الصلادة وناتج الخوارزمية الجينية يكون الحل المطلوب الذي يستخدم بالتضمين [15].

\section{3. تحويل Singular Values Decomposition (SVD)}

هي طريقة عددية تستخدم لتحليل المصفوفات. إن تحويل SVD

ثلاثة مصفوفات أخرى في نفس حجم المصفوفة الأصلية. في الجبر الخطي تعتبر الصورة عبارة عن صفوف ذات قيم عددية موجبة يمكن اعتبارها كمصفوفات. بثكل عام إذا كانت A هي مصفوفة مربعه

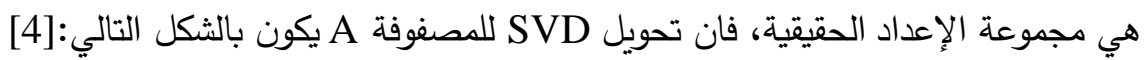
$\mathrm{A}=U S V^{T}$ مصفوفة متعامدة، و $V \in \mathrm{R}^{\mathrm{n} * \mathrm{n}} \quad \mathrm{U} \in \mathrm{R}^{\mathrm{n} * \mathrm{n}} \mathrm{U}$ مثل مصفوفة قطرية. $S=\left[\begin{array}{llll}\sigma_{1} & & & \\ & \sigma_{2} & & \\ & & \ddots & \\ & & & \sigma_{n}\end{array}\right]$



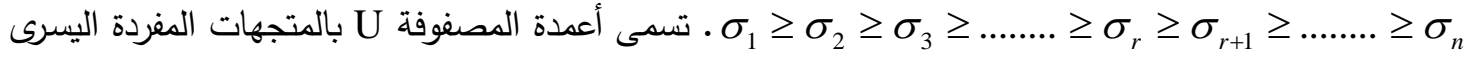
بينمـا تسمى أعمدة المصفوفة V ل بالمتجهات المفردة اليمنى للمصففوفة A. العناصـر القطريـة تمثل الإضــاءة Luminance بصورة عامة المصفوفة A لها عدة قيم فريدة والبعض منها صغير جدا وعدد القيم الفريدة غير الصفرية يساوي رتبة المصفوفة ADD . لها عدة خصائص. وحيث أن استخدامها في معالجة الصور الرقمية لها بعض 
الفوائد: أولها حجم المصفوفات الناتجة من عملية تحويل SVD غير ثابت ويمكن أن تكون مربعة أو مستطيلة.

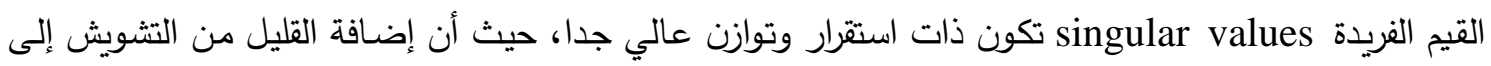



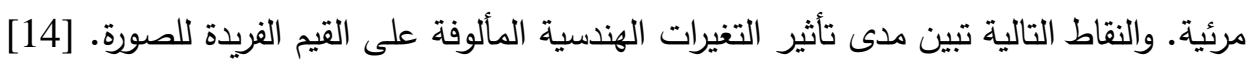
Transpose

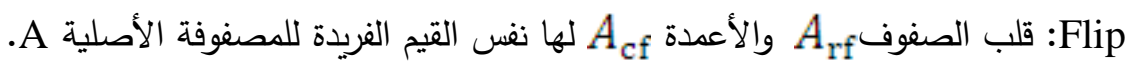
Rotation


المرات فان القيم الفريدة للمصفوفة الأصلية تساوي الأصلية. A A بإضـافة أعمدة أو صفوف صفرية إليها,فان المصفوفة الناتجة لها نفس القيم الفريدة غير الصفرية للمصفوفة الأصلية.[14] استتادا إلى هذه الخصائص فقد تم استخدام تحويلات SVD في طرق تضمين العلامة المائية. والفكرة الرئيسية تكمن في إيجاد القيم الفريدة Singular Values للغطاء المتمثلة بقطر المصفوفة S ومن ثم


في هذا البحث تم استخدام تحويل SVD في تضمين العلامة المائية وذلك بحساب المعدل للقيم الفريدة

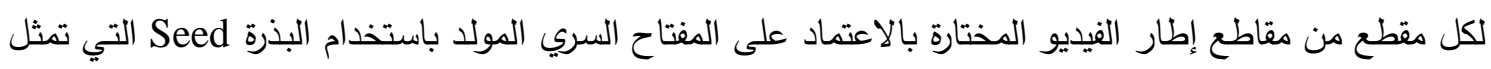

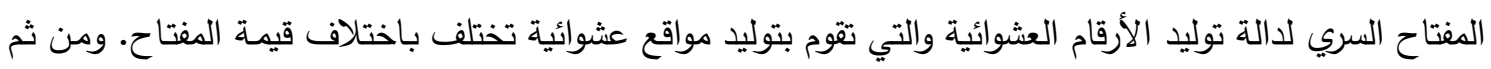
حساب الفرق بينه وبين قيمة المركز للمقطع المختار ومقارنته مع قيمة معامل الصلادة فإذا كانت قيمة الفرق أكبر من قيمة المعامل فسوف تأخذ قيمة المعدل للقيم الفريدة في عملية التضمين وإذا لا فيتم الاعتماد على قيمة مركز المقطع في التضمين حيث تم الحصول على نتائج مناسبة. أيضا تم استخدام طريقة SVD والمعدل مع الخوارزمية الجينية لتحسين الأداء والحصول على نتائج أفضل.

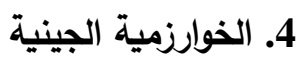

تتميز تقنية الخوارزميات الجينية عن التقنيات التقليدية السائدة بأن قوة هذه التقنية لا تكمن في قدرتها على التركيز لإيجاد الحلول الأمثلية الموضعية بل في اتساع نطاق المنطقة المدروسة المراد تمثيلها، وتعيين قيمة أمثلية

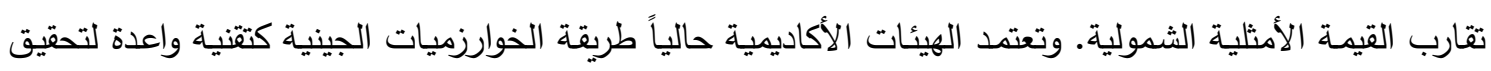

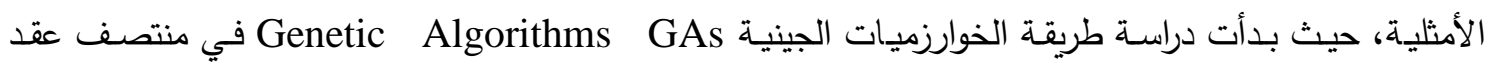

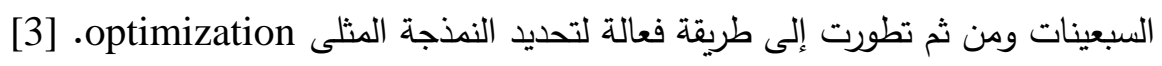




\section{4 الخوارزمية الجينية في نظام العلامة المائية}

تم توظيف الخوارزمية الجينية في نظام العلامة المائية، حيث يتم توليد مفاتيح عدة وباستخدام الخوارزمية الجينية يتم الحصول على الحل الأمثل أي بعد تتفيذ الخوارزمية الجينية على الجيل الابتدائي المكون من المفاتيح

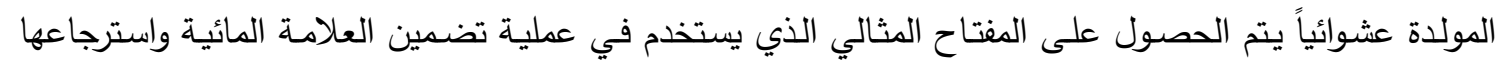
وذلك بعد اختيار وحساب دالة اللياقة المعتمدة على معامل الارتباط ومقياس الـ Peak Signal -to-Noise

[10] •Bit Correct Ratio BCR ومقياس Ratio PSNR

2.4 خطوات الخوارزمية

الخطوة الأولى: توليد d من المفاتيح بصورة عشوائية تمثل أفراد الجيل الابتدائي.

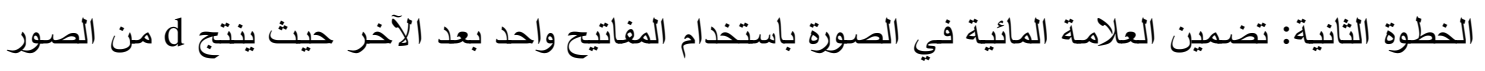
المضنة بها العلامة المائية.

الخطوة الثالثة: حساب قيمة PSNR لكل صورة متضمنة للعلامة المائية.

الخطوة الرابعة: تتفيذ الهجمات على كل الصور المتضمنة للعلامة المائية.

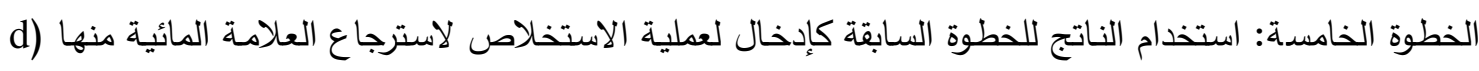

من العلامات المائية).

الخطوة السادسة: حساب قيمة BCR لكل فرد.

الخطوة السابعة: حساب دالة اللياقة لكل فرد.

الخطوة الثامنة: اختيار الفرد الذي يمتلك أعلى قيمة لياقة.

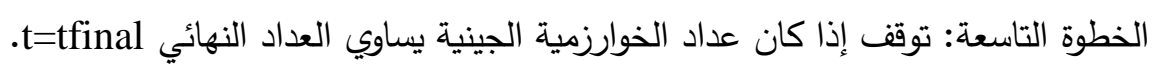
الخطوة العاشرة: تتفيذ عملية العبور لتوليد أفراد جدد للجيل القادم.

الخطوة الحادية عشر : تطبيق الطفرة الوراثية على كل فرد.

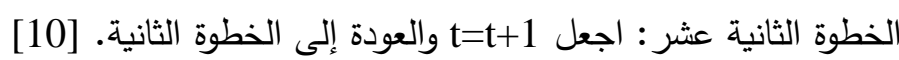

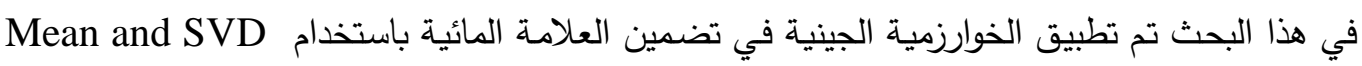

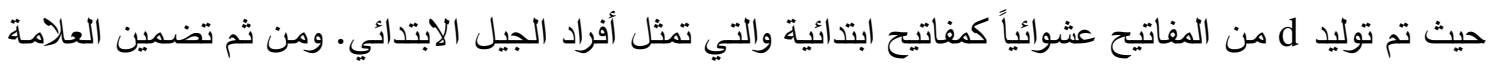

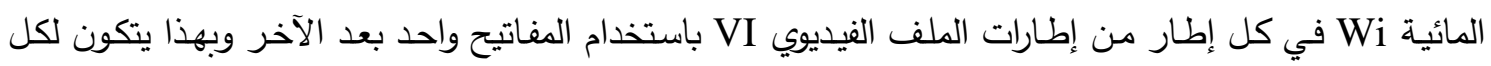

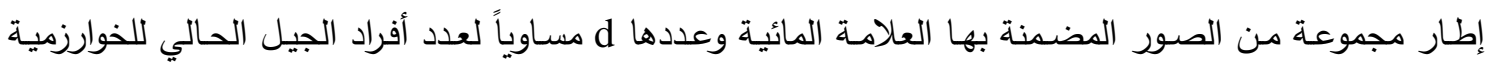

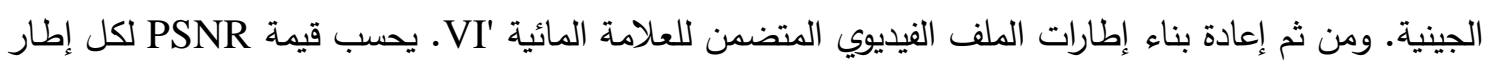
متضمن للعلامة المائية وتتفيذ الهجمات على كل إطار متضمن للعلامة المائية ومن ثم استخلاص العلامة المائية

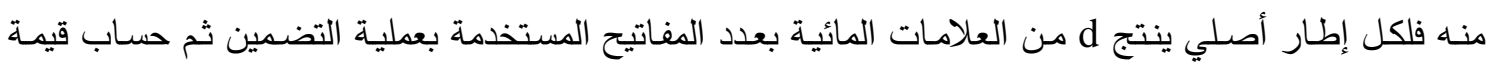

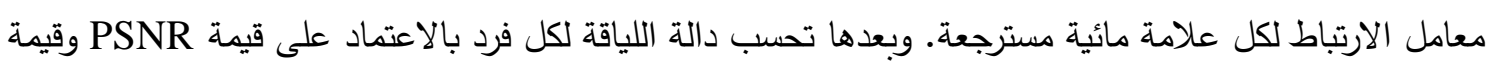
معامل الارتباط، وأخيراً يتم اختيار الفرد الذي يمتلك أعلى قيمة لياقة على مستوى الجيل الحالي الذي يمثل الذيل المواقع الأنسب لعملية التضمين. 
5. نظام العلامة المائية باستخدام الثبكات العصبية

ظهرت في السنوات الأخيرة العديد من البحوث التي استخدمت الشبكات العصبية لتصميم نظام تأشير

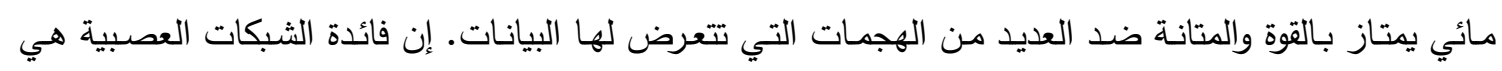
تحليل خصائص الصورة التي يتم تضمين العلامة المائية فيها حيث يتم التضمين فيها بالاعتماد على خصائص لئس

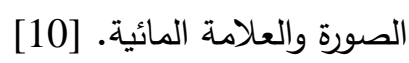

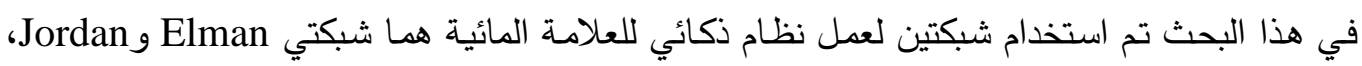
حيث تبدأ عملية تضمين العلامة المائية باختيار مقاطع من إطار الملف الفيديوي عددها يساوي عدد قيم العلامة

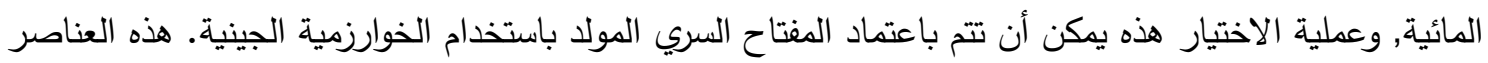

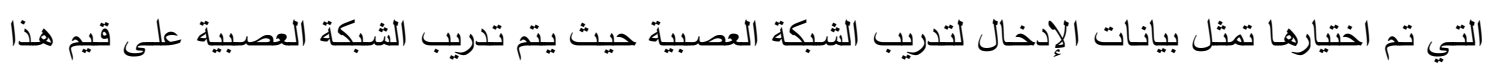
المقطع وان الإخراج المطلوب للشبكة العصبية هو النقطة المراد تضمين العلامـة المائية فيها والتي تمثل نقطسة المركز للمقطع الحالي. بعد تدريب الثبكة العصبية فان الإخراج هو قيمة قريبة جدا من القيمة المتوقعة. عملية

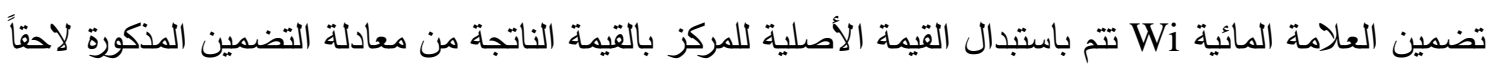

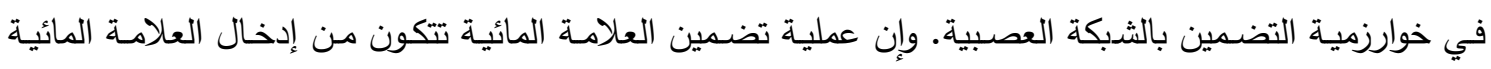

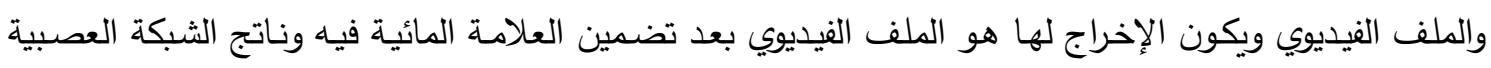

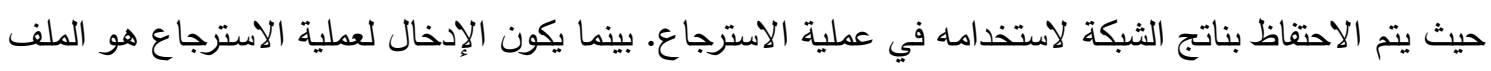

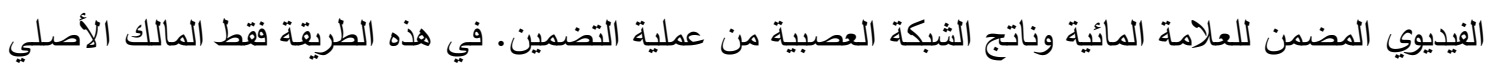

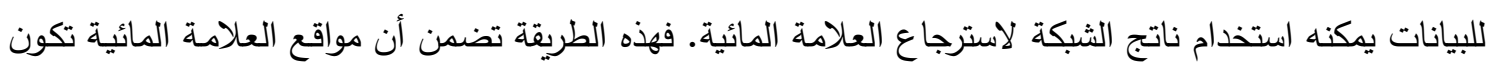
غير معروفة إلى إي جهة أخرى وبالتالي فقط الجهة الشرعية يمكنها استرجاع العلامة المائية من هذه البيانات.

Elman 1.5

تتألف شبكة Elman في الأقل من ثلاث طبقات من الخلايا: طبقة الإدخال، والطبقة الوسطى وتسمى

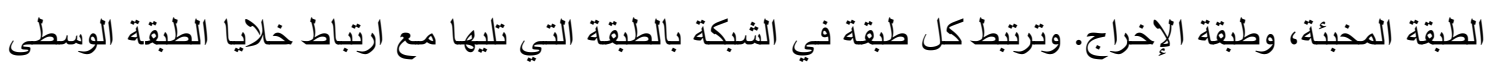

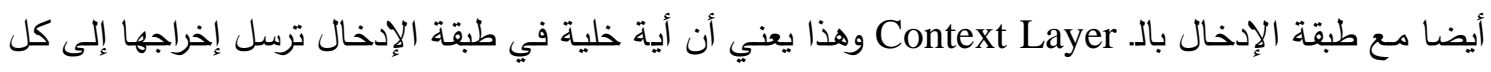

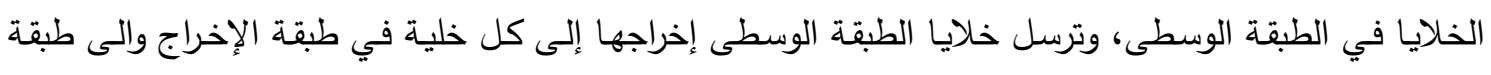

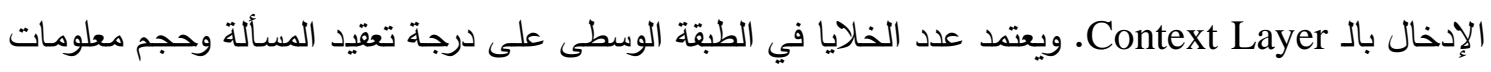
الإدخال. [9] ويوضح الثكل (1) الثبكة العصبية Elman المتكونة من ثلاث طبقات وهي طبقة الإدخال والطبقة الوسطى وطبقة الإخراج على الترتيب. 


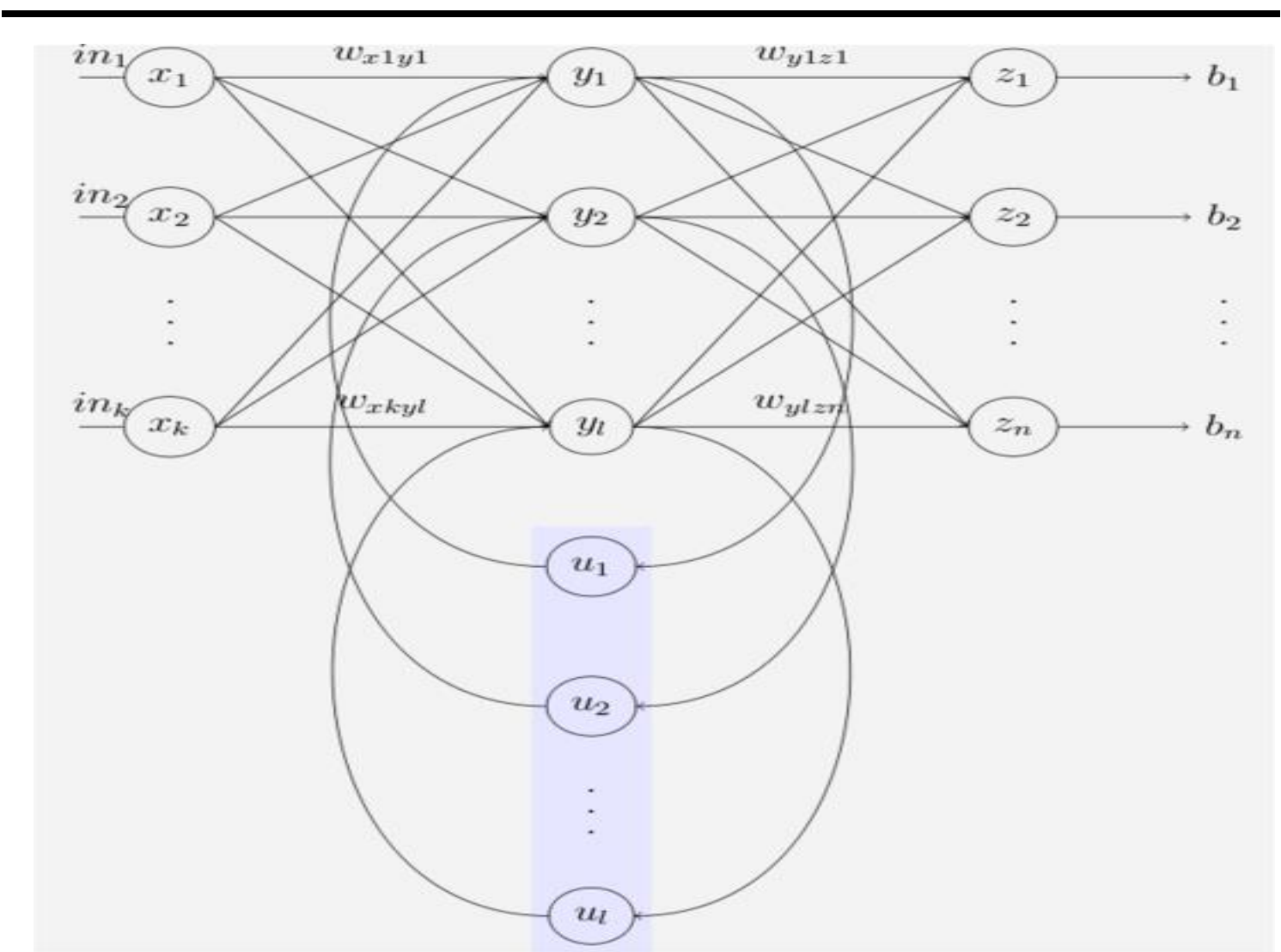

Elman الثكل (1). التركيب العام لشبكة

Jordan 2.5



[9].Hidden Layer إلى طبقة الإخراج Output Layer وليس من الطبقة الوسطى والثكل (2) يوضح التركيب العام للشبكة العصبية Jordan المتكونة من ثلاث طبقات وهي طبقة الإدخال والطبقة الوسطى وطبقة الإخراج على الترتيب.

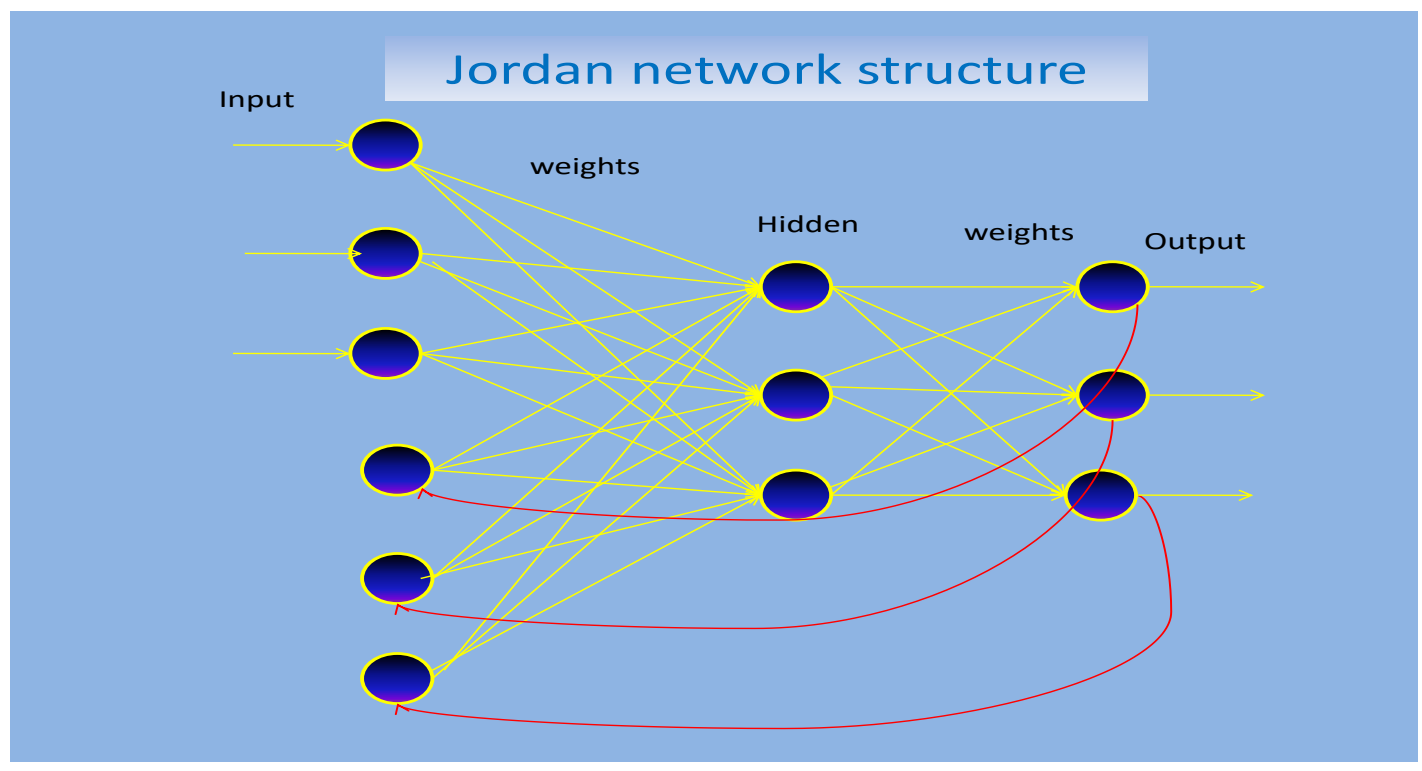

الثكل (2). التركيب العام لشبكة Jordan 
6. مقاييس الكفاءة

يتم حساب نسبة الخطأ بين الغطاء الأصلي والغطاء المتضمن العلامة المائية عن طريق حساب قيمة

Peak Signal -to-Noise Ratio PSNR

$$
\begin{aligned}
P S N R & =10 . \log _{10}\left(\frac{255^{2}}{M S E}\right) d B \\
M S E & =\frac{1}{n . n} \sum_{i=0}^{n-1} \sum_{j=0}^{n-1}(I(i, j)-I w(i, j))^{2}
\end{aligned}
$$

حيث إن I(i, j) يمثل الغطاء الأصلي و Iw(i, j) يمثل الغطاء المتضمن العلامة المائية. يجب أن تكون العلامة المائية المسترجعة مشابهة للعلامة المائية الأصلية ويتم حساب نسبة التطابق من خلال حساب معامل الارتباط Normalized Correlation Coefficient

$$
N C C=\frac{\sum_{i=1}^{M 1} \sum_{j=1}^{M 2} w(i, j) w^{*}(i, j)}{\sqrt{\sum_{i=1}^{M 1} \sum_{j=1}^{M 2} w(i, j)^{2} \sum_{i=1}^{M 1} \sum_{j=1}^{M 2} w^{*}(i, j)^{2}}}
$$

حيث إن w(i,j) تمثل العلامة المائية الأصلية وw(i,j) تمثل العلامة المائية المسترجعة.

\section{Mean and SVD أولاً عملية التضمين باستخذام}

تبدأ عملية تضمين العلامـة المائية في الملف الفيديوي بإدخال الملف الفيديوي والعلامـة المائية ثم يتت

فصل إطارات الملف الفيديوي ليتم تضمين العلامة المائية في كل إطار من إطارات الملف الفيديوي على حدة. يتم ذلك بقراءة الملف الفيديوي ذي الامتداد avi. ومن ثم أخذ كل إطار من إطارات الملف الفيديوي والتعامل معه على حدة حسب المقطع البرمجي التالي.

$\%$------------ read avi file and its information

[filename pathname]=uigetfile( $\left\{{ }^{* *}\right.$.avi';'***'\},'open vedio file');

info=aviinfo([pathname filename] $)$

avi=aviread([pathname filename]);

aviobj=avifile('a1.avi');

for index $=1$ :size $($ avi, 2$)$

frame=avi(index); \%-- read ith-frame from avi vedio

[imagframe,map] =frame $2 \mathrm{im}$ (frame);\%convert frame to rgb imageframe

ycbcr=rgb2ycbcr(imagframe);\%covert imageframe to ycbcr color space

orginal_frame $=$ double $(\mathrm{ycbcr}(:,:, 1)) ; \%$ select y matrix for embeding

watermarked_frame $=$ double(orginal_frame);

lengt $=32$;

for $i=1$ :lengt

for $j=1$ :lengt

$\mathrm{r}=\operatorname{keyr}(\mathrm{i}, \mathrm{j}) ; \mathrm{c}=\operatorname{keyc}(\mathrm{i}, \mathrm{j})$;

block_input=orginal_frame $(\mathrm{r}-1: \mathrm{r}+1, \mathrm{c}-1: \mathrm{c}+1)$;

[u s v] = svd(block_input);

meanofsvd $(\mathrm{i}, \mathrm{j})=$ mean $(\operatorname{diag}(\mathrm{s}))$;

$\mathrm{sub}=\operatorname{abs}($ meanofsvd $(\mathrm{i}, \mathrm{j})$ - orginal_frame $(\mathrm{r}, \mathrm{c}))$;

if (sub $>=$ strength)

value $1=$ meanofsvd $(i, j)$; 
else

$$
\text { value1 = orginal_frame }(\operatorname{keyr}(i, j), \operatorname{keyc}(i, j))
$$

end

if orginal_water $(\mathrm{i}, \mathrm{j})==1$ $\mathrm{x}=\max ($ value1,orginal_frame $(\mathrm{r}, \mathrm{c})+$ strength $)$;

else $\mathrm{x}=\min ($ value1,orginal_frame(r,c)-strength);

end

$$
\text { watermarked_frame }(r, c)=x \text {; }
$$

end

end

ycbcr(:,:,1)=watermarked_frame;

rgb=ycbcr2rgb(ycbcr);

Framepsnr(index $)=$ mean $(\operatorname{psnr}($ double $(\mathrm{rgb})$, double(imagframe) $))$;

frame $=$ im 2 frame(rgb,map);

aviobj=addframe(aviobj,frame);

$\operatorname{av}($ index $)=$ frame;

end

meansvd(index,1:1024)=meanofsvd(:)';

save('meansvd_key.mat','meansvd','keyr','keyc');

aviobj=close (aviobj);

avi1=aviread('a1.avi');

save avi3.mat av;

$\%$

يتم تحويل كل أطار من صيغة الـ RGB إلى صيغة الـ Ycbcr للألوان (هذه الصيغة ستحول الإطارات

الملونة إلى ثلاث طبقات هي Y وهي تمثل شدة إضـاءة الصورة، Cb التي تمثل التلوينة الزرقاء و Cr التي تمثل

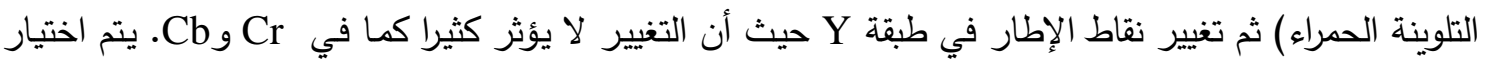
مقاطع بصورة عشوائية بعدد بتات العلامة المائية ويتم حساب قيم SVD لكل مقطع وتضمين العلامة المائية فيها ثم إعادة بناء المقاطع في مصفوفة Y ثم إعادة التحويل من YCbCr إلى الملف الفيديوي ليتم الحصول على الفيديو المضمن للعلامة المائية كما موضح ذلك بالمقطع البرمجي السابق.

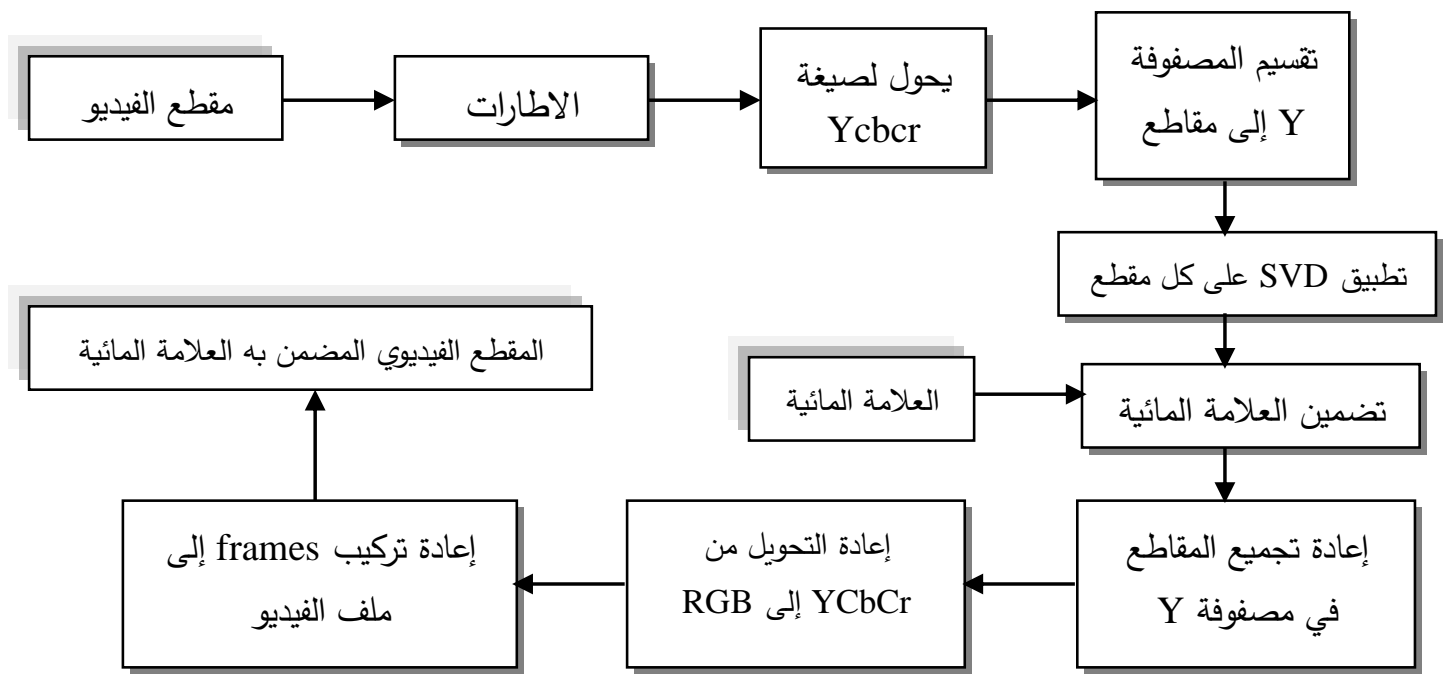

Mean and SVD الثكل (3). المخطط العام لتضمين العلامة المائية بطريقة 
الخطوة الأولى: قراءة الملف الفيديوي VI والعلامة المائية W ذات الحجم M*N.

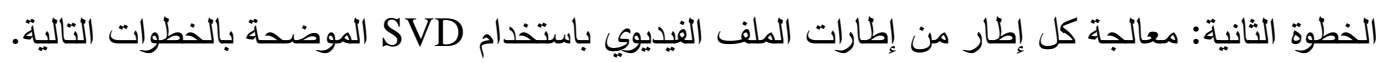

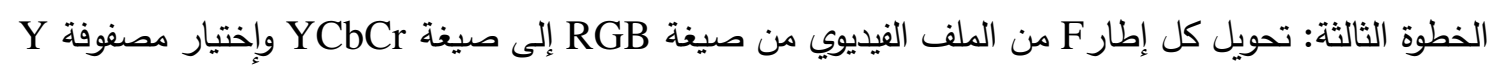

للتضمين داخلها.

الخطوة الرابعة: توليد مفتاح سري لتحديد المواقع ضمن الغطاء لتضمين العلامة المائية.

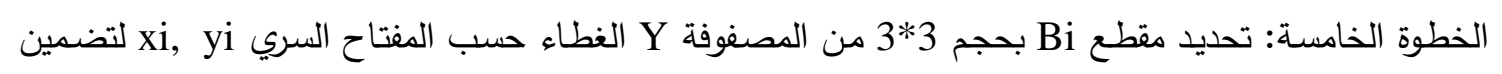

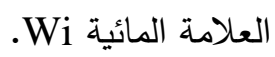

الخطوة السادسة: تطبيق Singular Values Decompositions SVD على قيم كل مقطع مختار Bi حيث $\mathrm{Bi}=\mathrm{USVT}$

أي أنه يتم تنفيذ الإيعاز التالي على كل مقطع من مقاطع الإطار المختارة اعتماداً على المفتاح

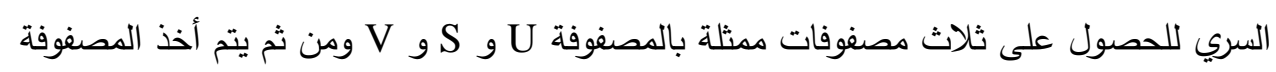
S والتعامل معها لإجراء عملية التضمين:

[U S V]= svd(block_input);

وإن Block_Input يمثل المقطع المختار ضـمن الإطـار المعين من إطـارات الملف الفيديوي المطلوب التضمين فيه.

الخطوة السابعة: حساب المعدل mean للقيم الفريدة الناتجة من الخطوة السابقة المثثلة بالمصفوفة S: Mean1=mean $(\operatorname{diagonal}(\mathrm{S}))$

الخطوة الثامنة: تضمين قيم العلامة المائية بالمراكز للمقاطع Bi المختارة حسب المفتاح السري كما يلي: $Y^{\prime}(x i, y i)=\left\{\begin{array}{lc}\max \{\text { value }, \text { center }(B i)+\delta\} & w i=1 \\ \min \{\text { value }, \text { center }(B i)-\delta\} & w i=0\end{array}\right.$

value $= \begin{cases}\text { mean } 1, & \mid \text { mean } 1-\operatorname{center}(B i) \mid>\delta \\ \operatorname{center}(B i), & \mid \text { mean } 1-\operatorname{center}(B i) \mid \leq \delta\end{cases}$ حيث أن:

wi تمثل بت من بتات العلامة المائية، بمثل الغطاء المتضمن العلامة المائية وتستمر عملية

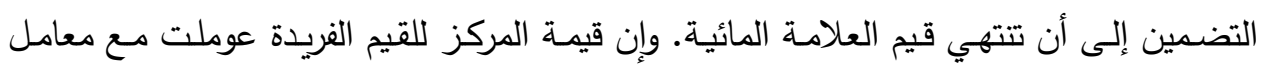

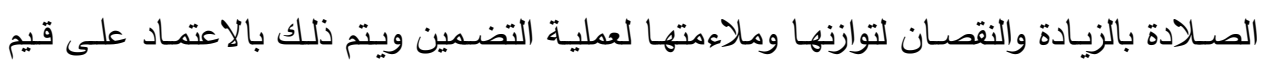

العلامة المائية وقيمة المتغير Value اعتمدت على الفرق مابين المعدل للقيم الفريدة والمركز . الخطوة التاسعة: تحويل إطار الملف الفيديوي'F'المتضمن للعلامة المائية من صيغة YCbCr إلى صيغة ثم إعادة بناء إطارات الملف الفيديوي المتضمن للعلامة المائية 'VI وعرضهنه. الخطوة العاشرة: حساب قيمة PSNR لجميع الإطارات المتضمنة العلامة المائية. 
Mean and SVD ثالثاً عملية الاسترجاع باستخدام

تبدأ هذه العملية بإدخال الملف الفيديوي المتضمن للعلامة المائية حيث يتم فصل إطارات الملف الفيديوي

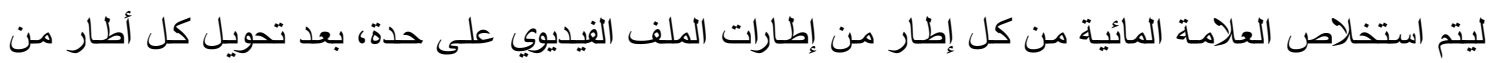
صيغة RGB إلى صيغة YCbCr للألوان كما مبين بالخوارزمية التالية:

الخطوة الأولى: قراءة الملف الفيديوي المتضمن للعلامة المائية 'VI'

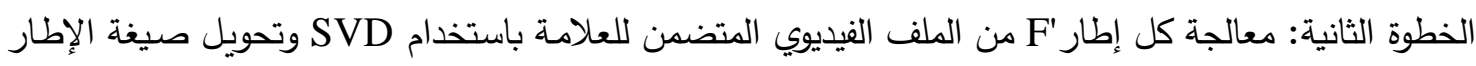
من RGB إلى YCbCr واختيار Y' لاستخلاص العلامة المائية باعتماد المفتاح السري.

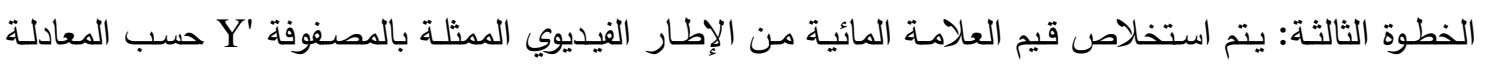

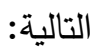

$$
W i=\left\{\begin{array}{l}
1, Y^{\prime}(x i, y i)>\text { meansvd }(I) \\
0, \text { otherwise }
\end{array}\right.
$$

وتستمر هذه العملية إلى أن يتم استرجاع جميع قيم العلامة المائية.

الخطوة الرابعة: حساب قيمة معامل الارتباط NCC للعلامة المائية المسترجعة.



Mean and SVD الثكل (4). المخطط العام لعملية استرجاع العلامة المائية بطريقة

8 خوارزمية التضمين باستخدام Mean and SVD المهنة ذكائياً خطوات تطبيق الخوارزمية الجينية على نظام العلامة المائية في تحويل SVD والمعدل لكل إطار من

إطارات الملف الفيديوي يمكن تلخيصها بالثكل التالي: الخطوة الأولى: توليد d من المفاتيح عشوائياً بصورة مباشرة دون الحاجة إلى استخدام البذرة كمفاتيح ابتدائية والتي تمثل أفراد الجيل الابتدائي. الخطوة الثانية: تضمين العلامة المائية Wi في كل إطار من إطارات الملف الفيديوي VI باستخدام المفاتيح واحد بعد الآخر (وذلك بعد تحديد مقطع Bi بحجم 3*3 من المصفوفة Y الغطاء حسب احد المفاتيح السرية ثم تطبيق SVD على قيم كل مقطع مختار Bi، ومن ثم حساب المعدل Mean وند للقيم

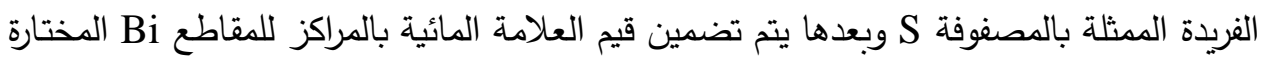
وذلك حسب المعادلة التالية: 


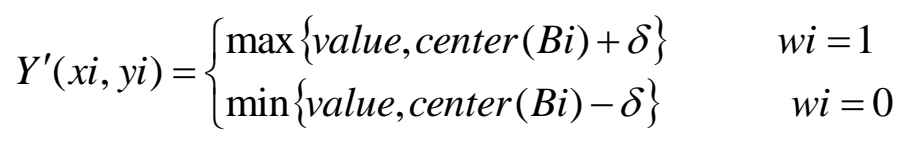

حيث Y' تمثل الغطلـاء المتضـمن للعلامـة المائيسة وتستمر عمليـة التضـمين إلى أن تتنهي قيم العلامة المائية. تحويل إطار الملف الفيديوي F' المتضمن للعلامة المائية من صيغة Ycbcr إلى

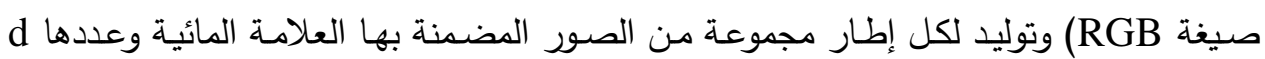
مساويا لعدد أفراد الجيل الحالي للخوارزمية الجينية. ومن ثم إعادة بناء إطارات الملف الفيديد الفيديوي

المتضمن للعلامة المائية 'VI.

الخطوة الثالثة: حساب قيمة PSNR لكل إطار متضمن للعلامة المائية لتقييم جودته. الخطوة الرابعة: تتفيذ الهجمات على كل إطار متضمن العلامة المائية ومن ثم استخلاص العلامة المائية منه فلكل



$$
\text { قيمة معامل الارتباط لكل علامة مائية مسترجعة. }
$$

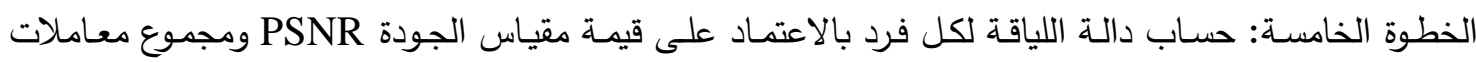

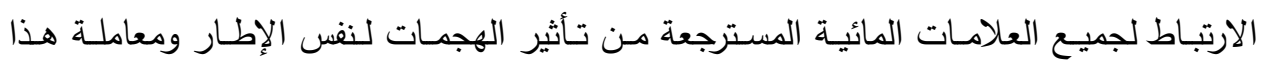

المجموع بمعامل الصلادة لموازنة العملية وذلك حسب المعادلة التالية:

$$
\text { fitness }(j)=\operatorname{PSNR}+\delta * \sum_{i=1}^{n} N C C\left(W, W_{i}^{\prime}\right)
$$

حيث j تمثل رقم الإطار المتضمن للعلامة المائية حسب المفتاح الأول من d من المفاتيح المولدة

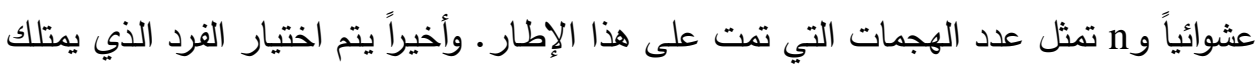
أعلى قيمة لياقة على مستوى الجيل الحالي الذي يمثل المواقع الأنسب لعملية التضدين.

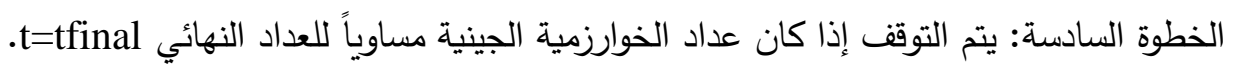
الخطوة السابعة: تتفيذ عملية العبور لتوليد أفراد جدد للجيل القادم، ومن ثم تطبيق الطفرة الوراثية. الخطوة الثامنة: زيادة عداد الخوارزمية الجينية t=t+1 والعودة إلى الخطوة الثانية. وأخيراً المفتاح الأفضل هو الذي يستخدم في عملية تضمين واسترجاع العلامة المائية.

9 9. عملية التضمين باستخدام الثبكات العصبية الاصطناعية

تشـبه عمليـة تضـمين العلامـة المائيسة في الملف الفيديوي باستخدام الثـبكات العصـبية الاصـطناعية خوارزمية Mean and SVD من حيث الهيكل العام لكن الاختلاف الأساسي هو أنه يتم تطبيق الثبكة العصبية الاصطناعية على المقاطع بدل تطبيق حساب القيم الفريدة SVD وبمفتاح سري مولد بالخوارزمية الجينية مهيأ مباشرة لعمل الشبكة وتوضح الأشكال (6, 5) المخطط العام لعملية تضمين واستخلاص العلامة المائية باستخدام الثبكات العصبية. 


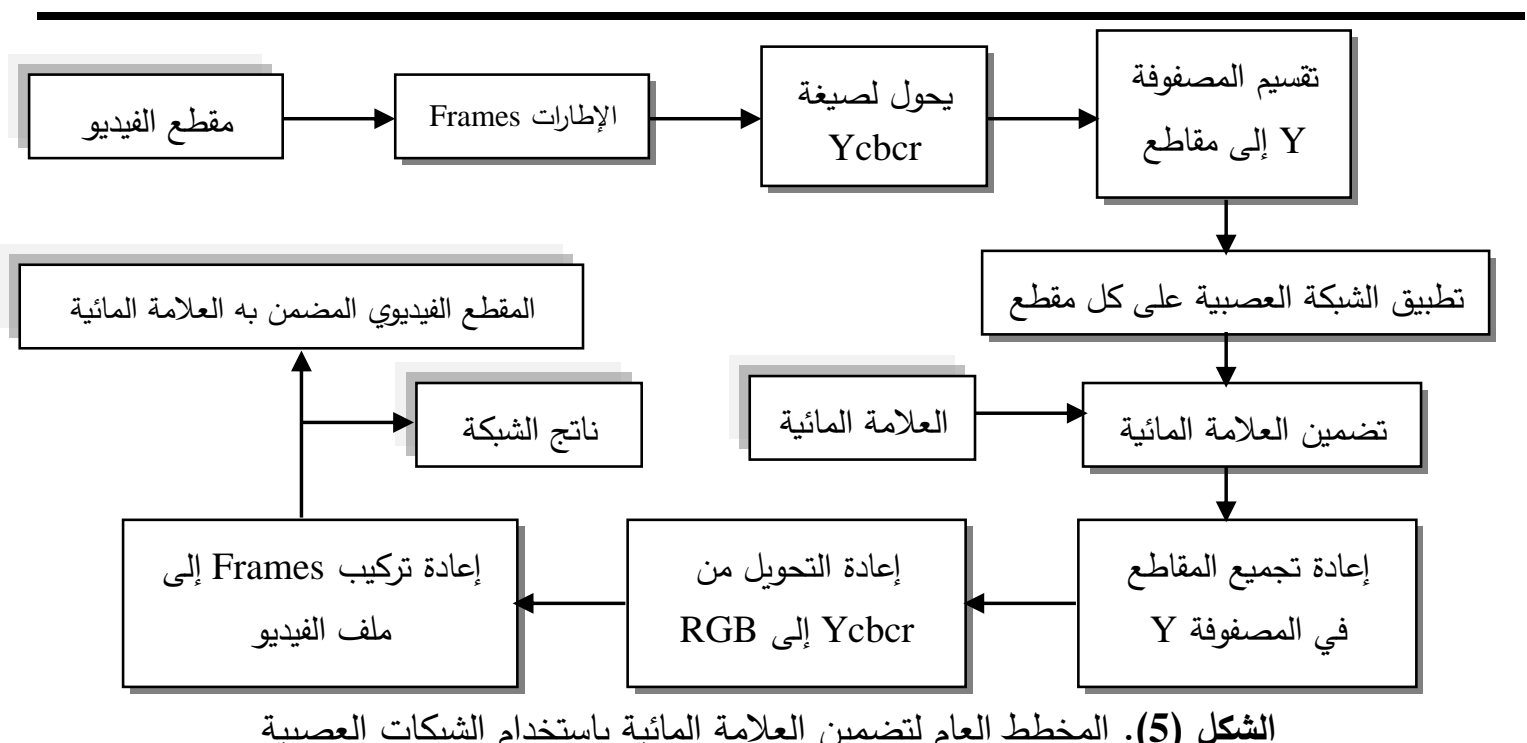

الثكل (5). المخطط العام لتضمين العلامة المائية باستخدام الثبكات العصبية

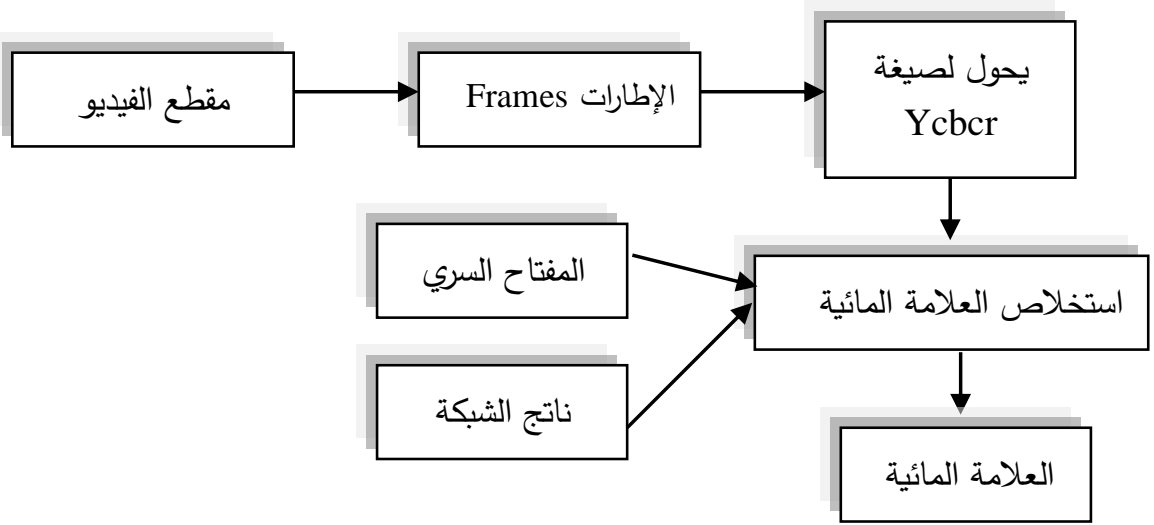

الشكل (6). المخطط العام لاسترجاع العلامة المائية باستخدام الثبكات العصبية

تضـمين العلامـة المائيـة Wi في كل إطـار مـن إطـارات الملف الفيديوي VI باستخدام المفتـاح السـري (المولد باستخدام الخوارزمية الجينية حيث تم توليد المفتاح أي المواقع التي يتم التضمين بها في كل مقطع من مقاطع

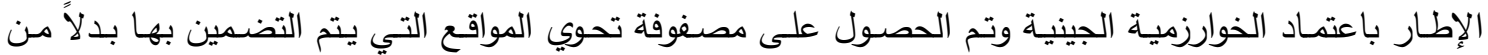
المفتاح المولد عشوائياً) وذلك بعد تحديد مقطع Bi بحجم 3*3 من المصفوفة Y الغطاء حسب المفتاح السري ثم إدخال بيانات هذا المقطع إلى الثبكة العصبية الاصطناعية Elman أو شبكة Jordan الموضح تدريبها بالمقطع

net.hidout $=0$;

for $i=1: 3$

for $j=1: 9$

net.hidnet $(j)=\operatorname{dot}($ net.input, net.wet $(\mathrm{j}, 1:$ len $))+$ net.wet $(\mathrm{j}, 11)$;

end \%net.input represent the value of block that selected

net.hidout=logsig(net.hidnet);

net.outnet $=\operatorname{dot}($ net.hidout, net.outwet);

net.out=logsig(net.outnet);

$\mathrm{dlt}=(\text { targ-net.out })^{*}(1 \text {-net.out })^{*}$ net.out;

net.outwet $=$ net.outwet + dlt. ${ }^{*}$ net.hidout ${ }^{*}$ beta;

for $\mathrm{j}=1: 9$

$s=$ net.outwet $(j) * d 1 t^{*}$ net.hidout $(j) *(1-$ net.hidout $(j))$; 
net.wet $(\mathrm{j}, 1:$ len $)=$ net.wet $(\mathrm{j}, 1:$ len $)+$ beta $^{*}$ s. ${ }^{*}$ net.input;

end

len $=10$;

net.input $(10)=$ net.outnet;

mse $=(\text { targ-net.outnet })^{\wedge} 2 / 2$;

if abs(mse) $<0.0001$

break;

end

end

$\mathrm{y}=$ net.outnet;

وبعدها يتم تضمين قيم العلامة المائية بالمراكز للمقاطع Bi المختارة وذلك حسب المعادلة التالية: $Y^{\prime}(x i, y i)=\left\{\begin{array}{lc}\max \{\text { value }, \text { center }(B i)+\delta\} & w i=1 \\ \min \{\text { value }, \text { center }(B i)-\delta\} & w i=0\end{array}\right.$

حيث أن:

(10) value $= \begin{cases}\text { netresult }, & \mid \text { netresult }- \text { center }(B i) \mid>\delta \\ \text { center }(B i), & \mid \text { netresult }- \text { center }(B i) \mid \leq \delta\end{cases}$

حيث ' تمثل الغطاء المتضمن للعلامة المائية وتستمر عملية التضمين إلى أن تتنهي قيم العلامة المائية. ومن ثم

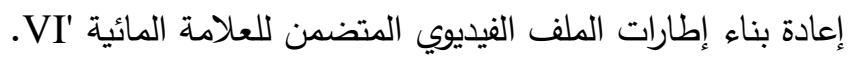

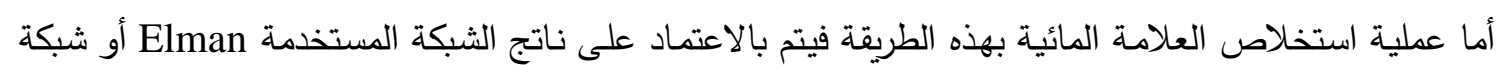
Jordan

10. 10 النتائج والمناقشة

تم تطبيق جميع طرائق البحث على الملف الفيديوي من نوعية (avi.) ذي الأبعاد 480*640 الذي يمثل الغطاء للعلامة المائية، وعدد الإطارات المكون منها الفيديو 101 إطار ـ أما بالنسبة للعلامة المائية فقد تم استخدام علامة مائية ثنائية Binary Image بأبعاد 32*32. وأثناء تتفيذ طرائق البحث على هذا الفيديو تم توجيه العديد من الهجمات للفيديو لاختبار قوة ومتانة طرائق البحث في تضمين العلامة المائية وكذلك في اختيار المفتاح الأمثل

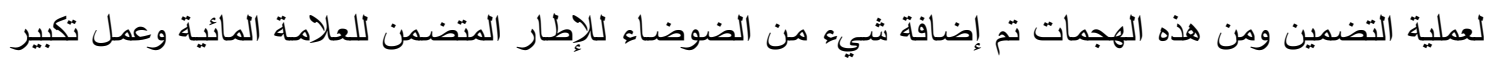

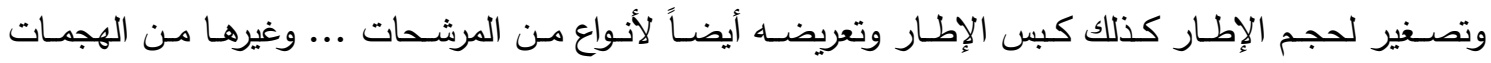
الموضحة نتائجها بالجداول والأشكال التالية.
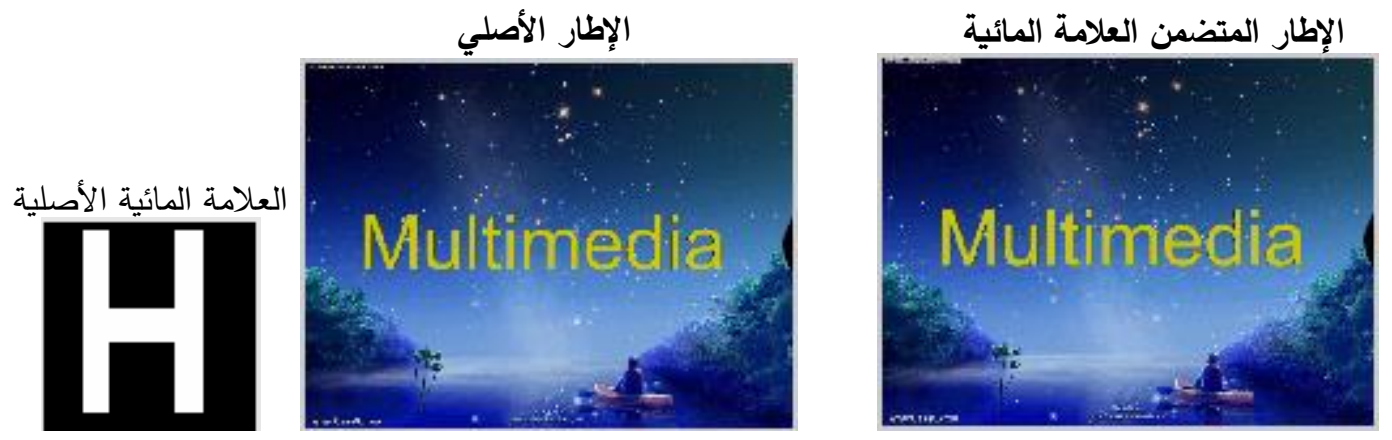

الثكل (7). يوضح الإطار الأصلي الأول والإطار المتضمن للعلامة المائية من الملف الفيديوي المستخدم بالبحث والعلامة المائية الأصلية المستخدمة 
جدول (1). يوضح قيم معامل الارتباط بين العلامة المائية الأصلية

والعلامة المائية المسترجعة بطريقة Mean and SVD

\begin{tabular}{|l|l|}
\hline \multicolumn{1}{|c|}{ Attacks } & NCC \\
\hline Salt\&pepper & 0.929 \\
\hline Gaussian & 0.910 \\
\hline Jpeg 50\% & 0.699 \\
\hline Jpeg 75\% & 0.873 \\
\hline Jpeg 90\% & 0.954 \\
\hline Lowpass & 0.870 \\
\hline Cropping & 0.699 \\
\hline Image resize & 0.732 \\
\hline sharpening & 0.922 \\
\hline blurring & 0.617 \\
\hline motion & 0.835 \\
\hline wiener & 0.923 \\
\hline
\end{tabular}

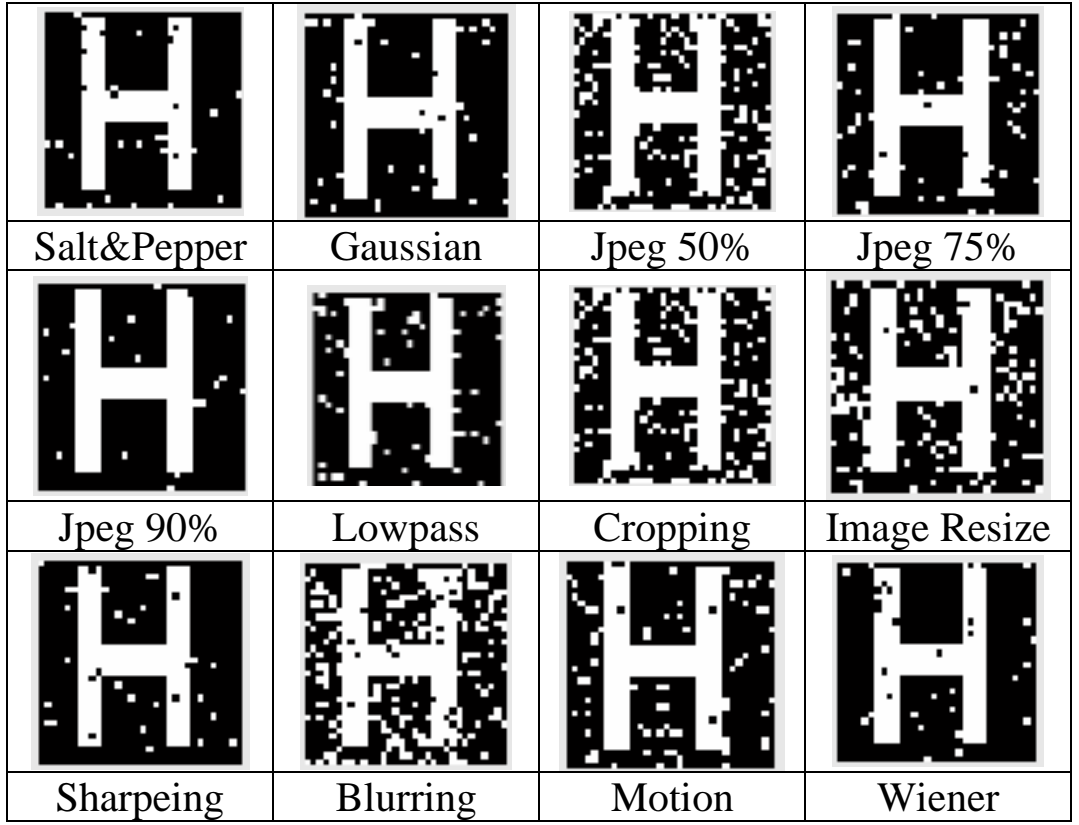

الثكل (8). يوضح العلامة المائية المسترجعة من الإطار المتضمن للعلامة المائية بطريقة Mean and SVD بعد تعريضه للهجمات

نلاحظ من الجدول والثكل السابقين أن طريقة mean and SVD التي طبقت على الفيديو أن نتائجها جيدة بعد توجيـه العديـ مـن الهجمـات حيث كانـت قيم معامـل الارتبـاط قريبـة مـن الواحد وهذا يعنـي أن العلامــة المائيـة

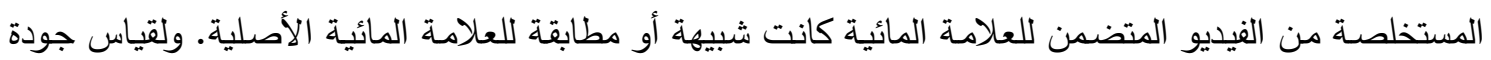
الطريقة المستخدمة تم كذلك قياس جودة الإطـار المتضمن للعلامـة المائية بمقياس PSNR حيث كانت قيمته 
الجدول (2). يوضح قيم معامل الارتباط بين العلامة المائية الأصلية والعلامة

المائية المسترجعة بطريقة mean and SVD باستخدام الخوارزمية الجينية

\begin{tabular}{|l|c|}
\hline \multicolumn{1}{|c|}{ Attacks } & NCC \\
\hline Salt\&Pepper & 0.951 \\
\hline Gaussian & 0.933 \\
\hline Jpeg 50\% & 0.811 \\
\hline Jpeg 75\% & 0.952 \\
\hline Jpeg 90\% & 0.997 \\
\hline Lowpass & 0.919 \\
\hline Cropping & 0.849 \\
\hline Image Resize & 0.829 \\
\hline Sharpening & 1 \\
\hline Blurring & 0.873 \\
\hline Motion & 0.870 \\
\hline Wiener & 0.963 \\
\hline
\end{tabular}

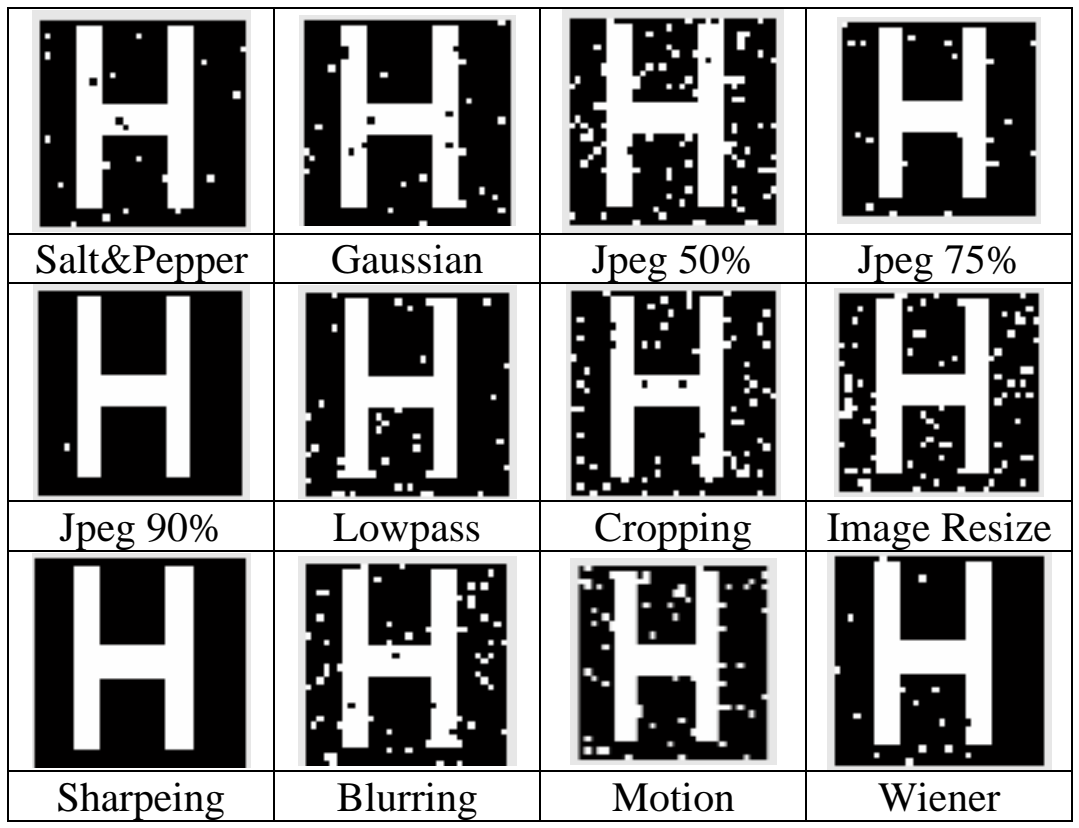

الثكل (9). يوضح العلامة المائية المسترجعة من الإطار المتضمن للعلامة المائية بطريقة

بean and SVD

بعد تطبيق طريقة mean and SVD المهجنة ذكائياً بالخوارزمية الجينية على الملف الفيديوي تم الحصول على نتائج أفضل من طريقة mean and SVD وهذا واضح من الجدول والثكل أعلاه حيث تم الحصول على على علامة مائية مطابقة للأصلية. إن نتائج الاختبارات أظهرت أن الخوارزمية المستخدمة في عملية التضمين والاسترجاع قوية ضد عملية الكبس باستخدام JPEG compression حتى في حالة استخدام معامل كبس واطئ. وإن قيمة 53.6942 dB هنا هي PSNR 
الجدول (3). يوضح قيم معامل الارتباط بين العلامة المائية الأصلية والعلامة المائية المسترجعة

بطريقة الثبكة العصبية Elman مع المفتاح السري المولد بالخوارزمية الجينية

\begin{tabular}{|l|c|}
\hline \multicolumn{1}{|c|}{ Attacks } & NCC \\
\hline Salt\&Pepper & 0.968 \\
\hline Gaussian & 0.995 \\
\hline Jpeg 50\% & 0.869 \\
\hline Jpeg 75\% & 0.995 \\
\hline Jpeg 90\% & 1 \\
\hline Lowpass & 0.930 \\
\hline Cropping & 0.869 \\
\hline Image Resize & 0.963 \\
\hline Sharpening & 1 \\
\hline Blurring & 0.928 \\
\hline Motion & 0.873 \\
\hline Wiener & 0.990 \\
\hline
\end{tabular}

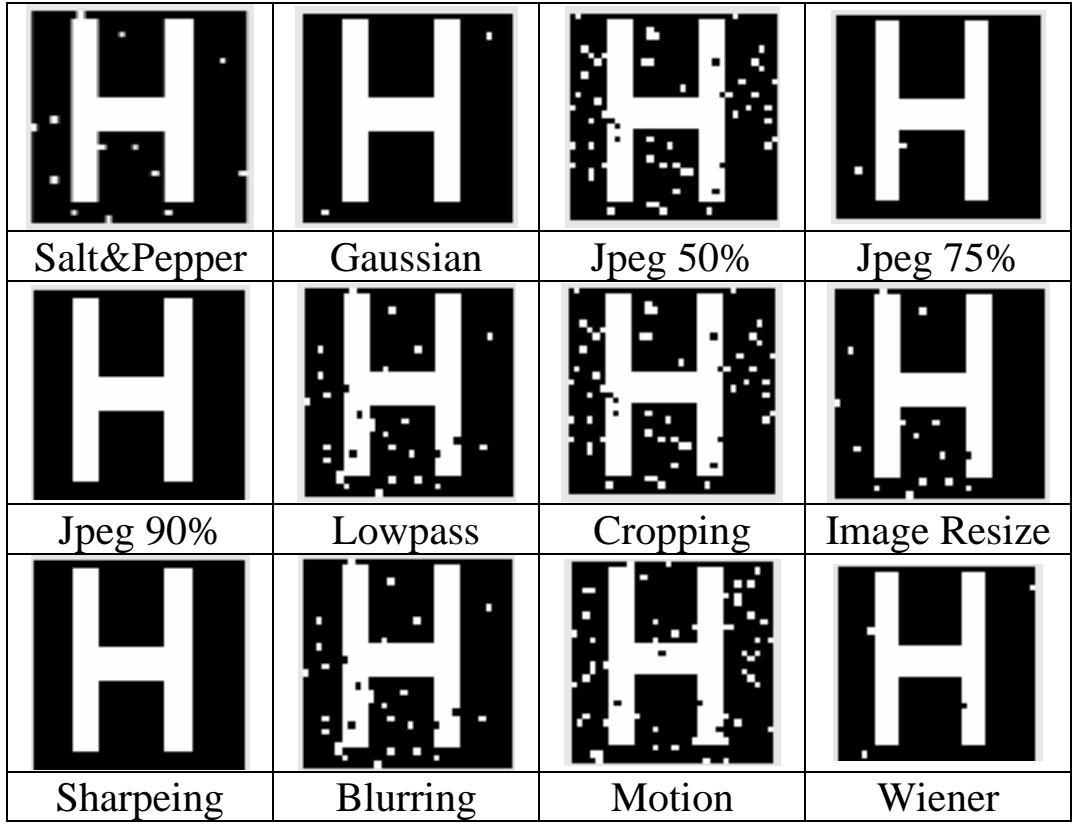

الشكل (10). يوضح العلامة المائية المسترجعة من الإطار المتضمن للعلامة المائية بطريقة الشبكة

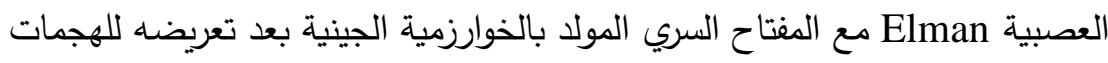

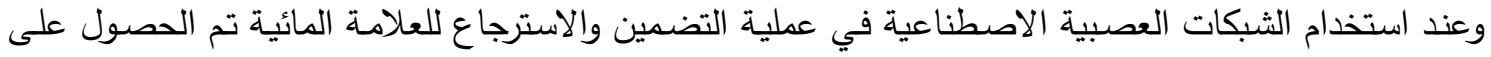
نتائج أفضل حيث تم الاعتماد على خصائص الغطاء والعلامـة المائية في عملية التضمين بالثبكات العصبية واستخدمت شبكتي elman و Jordan في تضمين العلامة المائية بالملف الفيديوي واستخدمت الخوارزمية الجينية هنا في توليد المفتاح السري فقط وتجهيزه لعملية التضمين وتم الحصول على نتائج مدتازة كما موضح بالجداول والأشكال الخاصة بالثبكات العصبية الاصطناعية حيث العلامة المسترجعة كانت مطابقة للعلامة الأصلية وقيم معامل الارتباط قريباً جداً من الواحد وهذا يؤيد كفاءة الطرائق المستخدمة بالبحث على الملف الفيديوي. إن النتائج 
أظهرت أن هذه الخوارزميـة قويـة ضد عملية الكبس باستخدام JPEG compression حتى في حالة استخدام

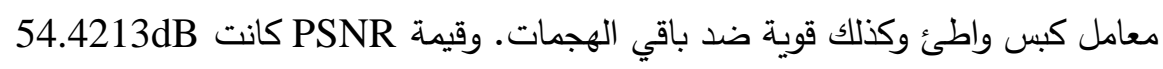
الجدول (4). يوضح قيم معامل الارتباط بين العلامة المائية الأصلية والعلامة المائية المسترجعة بطريقة الشبكة العصبية Jordan مع المفتاح السري المولد بالخو ارزمية الجينية

\begin{tabular}{|l|c|}
\hline \multicolumn{1}{|c|}{ Attacks } & NCC \\
\hline Salt\&Pepper & 0.968 \\
\hline Gaussian & 0.995 \\
\hline Jpeg 50\% & 0.869 \\
\hline Jpeg 75\% & 0.997 \\
\hline Jpeg 90\% & 1 \\
\hline Lowpass & 0.930 \\
\hline Cropping & 0.869 \\
\hline Image Resize & 0.963 \\
\hline Sharpening & 1 \\
\hline Blurring & 0.933 \\
\hline Motion & 0.883 \\
\hline Wiener & 0.990 \\
\hline
\end{tabular}

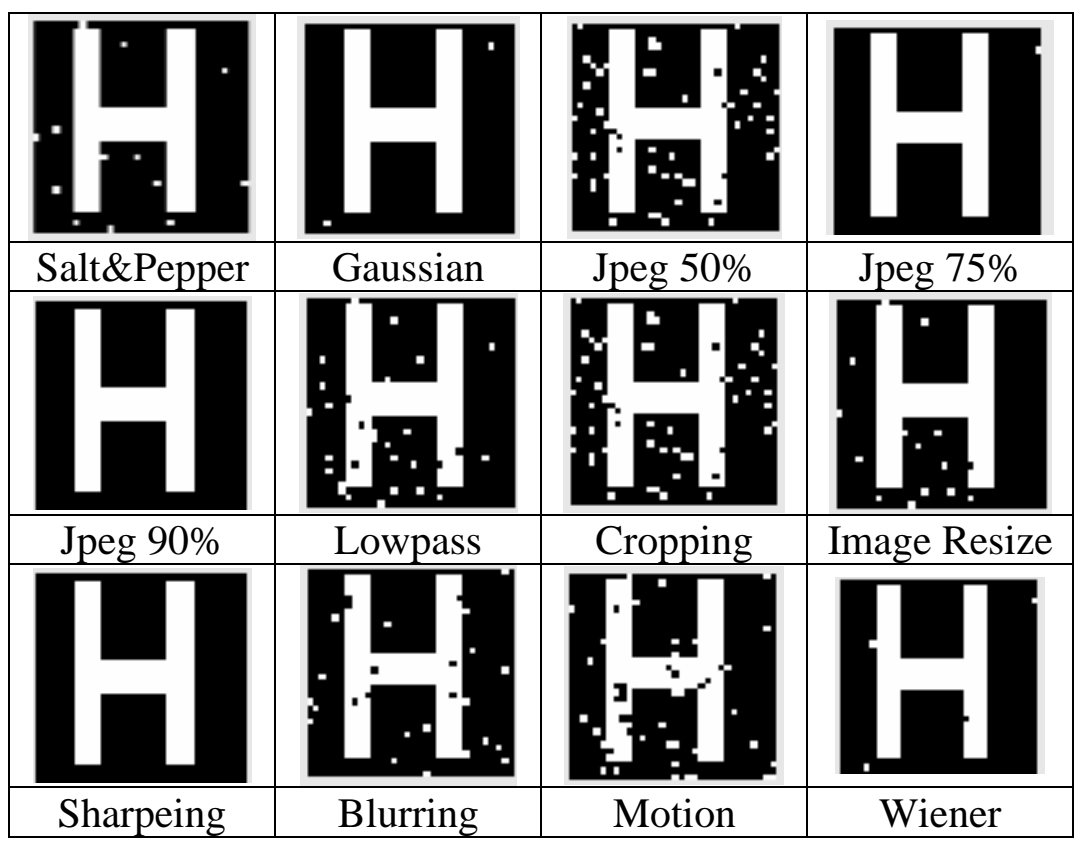

الثكل (11). يوضح العلامة المائية المسترجعة من الإطار المتضمن للعلامة المائية بطريقة الثبكة العصبية Jordan مع المفتاح السري المولد بالخوارزمية الجينية بعد تعريضه للهجمات الإنهات

11. الاستنتاجات

تعتبر الطرائق المستخدمة بالبحث لتضمين العلامة المائية من الطرائق القوية حيث: تميزت الخوارزميات المستخدمة بالقوة والمتانة تجاه العديد من الهجمات التي تتعرض لها لهن العلامدة المائية بقصد تغييرها أو إزالتها. 
كما تميزت العلامة المائية المضمنة بالملف الفيديوي بكونها غير مدركة في الإطار المضمن وتضمينها لا يسبب تغير أو تثوه في الغطاء الحامل أي الملف الفيديوي لها.

تتميز هذه الخوارزميات بكونها ذكائية لاستخدام الثبكات العصبية الاصطناعية والخوارزمية الجينية في تضمين العلامة المائية.

إن قوة العلامة المائية تتتاسب طرديا مع قيمة معامل صلادة العلامة المائية في معادلة التضمين أما جودة الإطار المضمن للعلامة تتتاسب عكسيا مع هذا المعامل.

تم الحصول على قيم دمتازة لمعامل الارتباط NCC التي أثبتت جودة وكفاءة الطرائق المستخدمة بالبحث. وكذلك قيم PSNR للإطار المضمن للعلامة المائية لجميع الطرائق المستخدمة. 


\section{REFERENCES}

$$
\begin{aligned}
& \text { الحمـامي، عـلاء حسن والحمامي، محمد عـلاء، "إخفاء المعلومـات: الكتابـة المخفيـة والعلامـة المائيـة"، } \\
& \text { مكتبة جامعة الثارقة، إثراء للنشر والتوزيع، } 2008 . \\
& \text { حامـد، فرقد، "توثيـق النصـوص باسـتخدام العلامـة المائيـة"، رسـالة ماجسـتير / كليـة علـوم الحاسـبات } \\
& \text { والرياضيات / جامعة الموصل، } 2004 .
\end{aligned}
$$

عادل، ندى وقنطنطين، مايا، "الخوارزميات الجينية"، جامعة تشرين / كلية هندسة المعلوماتية، 2009.

[4] Bedi S.S., Kumar A., and Kapoor P., "Robust Secure SVD Based DCT - DWT Oriented Watermarking Technique for Image Authentication". International Conference on IT to Celebrate S. Charmonman's 72nd Birthday, March, Thailand, pp. 46.1:46.7, 2009.

[5] El'arbi M., Amar C.R. and Nicolas H., "Video Watermarking Based On Neural Network", Ieee, Pp. 1577-1580, 2006.

[6] El'arbi M. , Amar C.R. and Nicolas H., "Avideo Watermarking Scheme Resistant To Geometric Transformations", Ieee, Pp. V-481: V-484, 2007.

[7] Ghazy R.A., El-fishawy N.A., Hadhoud M.M., Dessouky M.I. and Abd El-samie F.E., "An Efficient Block By Block Svd Based Image Watermarking Scheme", Ubiquitous Computing and Communication Journal, 2007.

[8] H. Seddik, M. Sayadi, F. Fnaiech, and M. Cheriet, "New Multi-Domains Image Watermarking Method Based On Multi-Watermarks Embedding And Neural Network Segmentation, Applied mathematical sciences, vol. 1, no. 39, pp. 19271939, 2007.

[9] Online: http://en.wikipedia.org/wiki/Recurrent_neural_network

[10] Jeng-Shyang Pan, Hsiang-Cheh Huang and Lakhmi C. Jain," Intelligent Watermarking Techniques", World Scientific Publishing Co. Pte. Ltd.,2004.

[11] Lee S., Jang D. and Yoo C., "An Svd Based Watermarking Method For Image Content Authentication With Improved Security", IEEE, pp. II-525: II-528, 2005.

[12] M. Ketcham, and S. Vongpradhip, "Intelligent Audio Watermarking Using Genetic Algorithm In DWT Domain", International Journal of Intelligent technology, vol. 2, no. 2, pp. 135-140, 2007.

[13] M. Shen, X. Zhang, L. Sun, P. Beadle and F. Chan, "A Method For Digital Image Watermarking Using ICA",4th International Symposium On Independent Component Analysis And Blind Signal Separation(ICA2003), 2003, Nara, Japan.

[14] S.M. Rafizul Haque, "Singular Value Decomposition And Discrete Cosine Transform Based Image Watermarking". Master Thesis, January 2008.

[15] Shieh C., Huang H., Wang F., and Pan J., "Genetic Watermarking Based On Transform Domain Techniques", Journal Of The Pattern Recognition Society, pp. 555-565, 2004.

[16] Wei Z., Li H., Dai J. and Wang S., "Image Watermarking Based On Genetic Algorithm", IEEE, pp. 1117-1120, 2006. 